\title{
Studies on the multiphoton ionization spectrum of the jet-cooled acetyl radical at $6.2-7.6 \mathrm{eV}$
}

\author{
Run-Hua $\mathrm{Li}^{\mathrm{a}, \mathrm{b}, *}$, Jen-Chien $\mathrm{Wu}^{\mathrm{c}}$, Jia-Lin Chang ${ }^{\mathrm{b}}$, Yit-Tsong Chen ${ }^{\mathrm{b}, \mathrm{c}}$ \\ a State Key Laboratory of Ultrafast Laser Spectroscopy, Zhongshan University, Guangzhou 510275, China \\ ${ }^{\mathrm{b}}$ Institute of Atomic and Molecular Sciences, Academia Sinica, P.O. Box 23-166, Taipei 106, Taiwan, ROC \\ ' Department of Chemistry, National Taiwan University, Taipei 106, Taiwan, ROC
}

Received 23 May 2001

\begin{abstract}
Acetyl radical was produced in a molecular beam by pyrolysis of acetone with a flash pyrolysis nozzle and was cooled by supersonic expansion. A continuous multiphoton ionization (MPI) spectrum of the jet-cooled acetyl radical at 6.2-7.6 eV was first observed in the experiment. The spectrum from $6.2 \mathrm{eV}$ to ionization threshold has resulted from $2+1$ resonance-enhanced MPI (REMPI) through its high-lying electronic states. These states are shown to be dissociative and metastable. The adiabatic ionization potential of the acetyl radical was redetermined to be $7.01 \pm 0.02 \mathrm{eV}$ from the derivative of MPI spectrum near ionization threshold directly. This work demonstrates that MPI technique can be easily used to monitor the produced acetyl radical in molecular beams. (C) 2001 Published by Elsevier Science B.V.
\end{abstract}

Keywords: Multiphoton ionization; Acetyl radical; Adiabatic ionization potential

\section{Introduction}

Acetyl radical $\left(\mathrm{CH}_{3} \mathrm{CO}\right)$ is one of the important intermediate species in combustion processes and in atmospheric reactions [1,2]. Studies on the electronic states, spectra and the dissociation dynamics of this radical are very helpful for combustion and atmospheric chemistry. The photoelectron spectra and ESR spectra have determined the structure of the acetyl radical in the

\footnotetext{
${ }^{*}$ Corresponding author. Tel.: +86-20-8403-7563; fax: +86-208403-7423.

E-mail address: stils04@zsu.edu.cn (R.-H. Li).
}

ground state $\left(\mathrm{X}^{2} \mathrm{~A}^{\prime}\right)[3,4]$. Theoretical and experimental studies on the ground state demonstrated that it is easy to dissociate to $\mathrm{CH}_{3}$ and $\mathrm{CO}$ with a low dissociation barrier, $\sim 17 \mathrm{kcal} / \mathrm{mol}$ [5-10]. Infrared spectra of the acetyl radical were also studied in matrix at low temperature by Bennett et al. [11] and by Jacox [12] in the ground state.

For the excited electronic states, theoretical calculations have been done to determine the energies of the four excited electronic states which are $60,113,154$ and $161.4 \mathrm{kcal} / \mathrm{mol}$, respectively $[13,14]$. Experimentally, Noda et al. [15] observed a continuous absorption spectrum of $\gamma$-irradiated $0.5 \mathrm{~mol} \% \mathrm{CH}_{3} \mathrm{COCl}$ at $77 \mathrm{~K}$ from 280 to $700 \mathrm{~nm}$. They assigned this band as the absorption of the 
acetyl radical. However, after the sample was illuminated with visible light, absorption in the visible region disappeared. UV absorption spectra in the range 200-280 $\mathrm{nm}$ were also observed at room temperature with different spectroscopic techniques [16-18]. However, the spectra of high states lying up $50000 \mathrm{~cm}^{-1}(6.2 \mathrm{eV})$ of the acetyl radical have still not been reported. Both the continuous absorption spectra and the photodissociation experimental studies $[19,20]$ demonstrate that the acetyl radical is very dissociative in the $\mathrm{UV}$ visible spectral region. The spectrum up $6.2 \mathrm{eV}$ of the acetyl radical is still blank until this date.

In this work, we prepared the acetyl radical in a molecular beam with a flash pyrolysis nozzle and first observed a continuous multiphoton ionization (MPI) spectrum at $6.2-7.6 \mathrm{eV}$. The mechanism of producing this spectrum was analyzed.

\section{Experiment}

The experimental setup for MPI and TOF mass spectrometer in our laboratory was described in detail in an earlier publication [21]. Briefly, a tunable dye laser (Lambda Physik, Scanmate 2E) pumped by an Nd:YAG laser (Spectra Physics, Quanta Ray GCR-190) was operated at $30 \mathrm{~Hz}$ with a pulse duration of $\sim 7 \mathrm{~ns}$. The dye laser was frequency-doubled with BBO or KDP crystals. Optogalvanic spectra of Ar were used to calibrate the fundamental frequency of the dye laser with $2 \mathrm{~cm}^{-1}$ accuracy. The laser beam was focused by a $25 \mathrm{~cm}$ focal length spherical lens and was aligned to intersect the molecular beam. A digital pulse/delay generator (SRS, DG535) was used to control the time sequence for the laser and molecular beams. The cations produced via MPI were repelled in an electric field with a direction perpendicular to both molecular and laser beams. The cations flew across a field-free TOF tube $(80 \mathrm{~cm})$ and were detected by a microsphere plate (MSP) (EI-MUI Technologies). The output signals of MSP were amplified by a preamplifier (EG\&G, VT120) and then were sent to a $500 \mathrm{MHz}$ digital oscilloscope (LECroy 9344) and a gated integrator (SRS, SR250) simultaneously. A personal computer was used to collect and process the experimental data after $\mathrm{A} / \mathrm{D}$ conversion.
The acetyl radical was produced by pyrolysis of acetone at about $1000{ }^{\circ} \mathrm{C}$. The precursor, acetone, was seeded in $2 \mathrm{~atm} \mathrm{He}$ with $0.1 \%$ concentration and was sprayed out by a pulsed valve. Before free expansion, it was pyrolyzed to methyl and acetyl radicals within an $8 \mathrm{~mm}$ length and $1 \mathrm{~mm}$ inner diameter resistance heated ceramic tube that was mounted on the nozzle of the pulsed valve. This flash pyrolysis nozzle was described in detail in [22]. The supersonic expanded radicals fly to the ionization region passing through a skimmer. The vertical distance from the nozzle to the ionization region is 9 $\mathrm{cm}$ to ensure that the radicals cool enough.

\section{Results}

\subsection{Production and detection of the acetyl radical}

In TOF mass spectra, both $\mathrm{CH}_{3}^{+}$and $\mathrm{CH}_{3} \mathrm{CO}^{+}$ came from two channels: the first one, acetone was photodissociated or pyrolyzed to $\mathrm{CH}_{3}$ and $\mathrm{CH}_{3} \mathrm{CO}$ firstly and then was ionized by the laser, that is

$$
\begin{aligned}
& \mathrm{CH}_{3} \mathrm{COCH}_{3} \stackrel{\text { hv/heat }}{\rightarrow} \mathrm{CH}_{3} \mathrm{CO}+\mathrm{CH}_{3} \\
& \mathrm{CH}_{3} \mathrm{CO} \stackrel{\text { ionization }}{\rightarrow} \mathrm{CH}_{3} \mathrm{CO}^{+} \\
& \mathrm{CH}_{3} \stackrel{\text { ionization }}{\rightarrow} \mathrm{CH}_{3}^{+}
\end{aligned}
$$

The second one, acetone was first ionized by laser because acetone cations are unstable, they fast decompose to $\mathrm{CH}_{3}^{+}$and $\mathrm{CH}_{3} \mathrm{CO}$ or $\mathrm{CH}_{3}$ and $\mathrm{CH}_{3} \mathrm{CO}^{+}$.

$$
\begin{aligned}
& \mathrm{CH}_{3} \mathrm{COCH}_{3} \stackrel{\text { ionization }}{\rightarrow} \mathrm{CH}_{3} \mathrm{COCH}_{3}^{+} \\
& \mathrm{CH}_{3} \mathrm{COCH}_{3} \stackrel{\text { decompose }}{\rightarrow} \mathrm{CH}_{3}+\mathrm{CH}_{3} \mathrm{CO}^{+} \\
& \mathrm{CH}_{3} \mathrm{COCH}_{3} \stackrel{\text { decompose }}{\rightarrow} \mathrm{CH}_{3}^{+}+\mathrm{CH}_{3} \mathrm{CO}
\end{aligned}
$$

In experiment, under the condition of heat on, we observed a significant $\mathrm{CH}_{3} \mathrm{CO}^{+}$signal within the wavelength range 330-380 $\mathrm{nm}$. However, under the condition of heat off, the $\mathrm{CH}_{3} \mathrm{CO}^{+}$signal disappeared. Fig. 1 shows the TOF mass spectra excited at $340 \mathrm{~nm}$. Comparing the signal of $\mathrm{CH}_{3} \mathrm{CO}^{+}$ under the condition of heat on to that under the condition of heat off, it can be concluded that the 


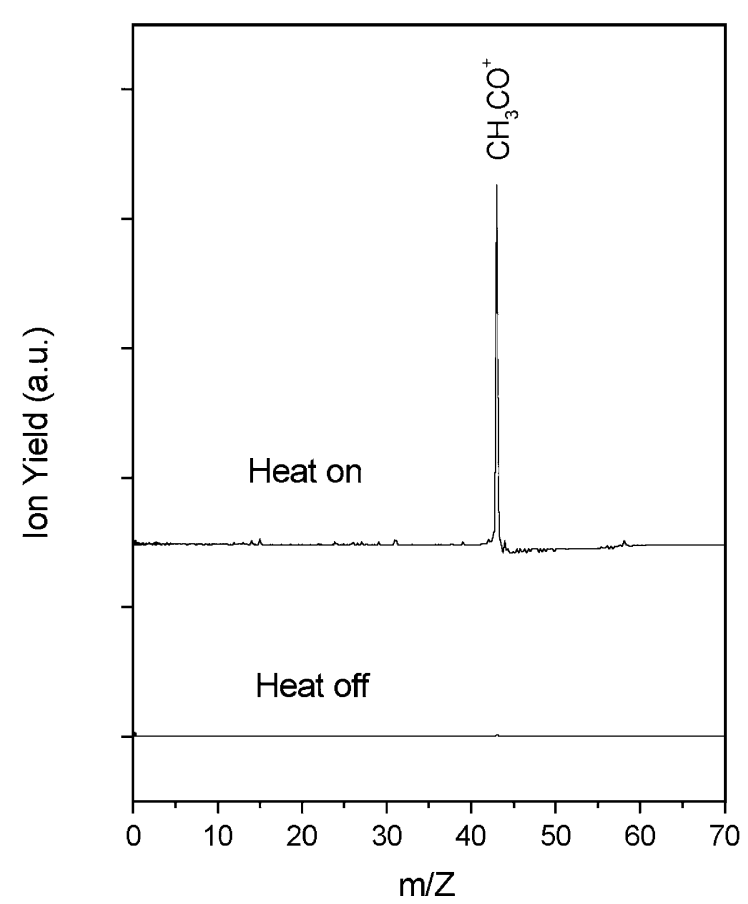

Fig. 1. Time-of-flight mass spectra of the acetyl radical excited at $340 \mathrm{~nm}$.

acetyl cations are produced from the first channel, not the second channel. That is to say, in our experiment, the acetyl radical was produced with pyrolysis of acetone and was detected with the MPI technique. As analyzed in Section 3.2, the acetyl radical is ionized via absorbing two photons simultaneously at this wavelength, not by stepwise $1+1$ MPI. This is the first time of detecting an acetyl radical with nanosecond pulsed laser with the MPI technique. Before this time, Abou-Zied and McDonald [23] have already detected the acetyl radical with picosecond pulsed laser with MPI technique in the experiment for studying the unimolecular photodissociation of $\mathrm{CH}_{3} \mathrm{CHO}$.

\subsection{MPI spectrum of the acetyl radical}

With the advantage of using dye laser with high pulse energy and high resolution in comparison with traditional UV light source, we try to find the vibronic spectrum of the acetyl radical in the range 200-250 nm (4.96-6.2 eV) by $1+1$ resonance-enhanced MPI (REMPI). In this scheme, the laser beam was slightly defocused. However, no $\mathrm{CH}_{3} \mathrm{CO}^{+}$signal was observed in the experiment under the condition of heat on. This is in agreement with the dissociative characterization of the acetyl radical in the UV spectral region.

We use $2+1$ REMPI to study the highly excited electronic states of the acetyl radical lying upon $6.2 \mathrm{eV}$. In this scheme, the laser beam is tightly focused with a spherical lens to the ionization region. In the range $325-400 \mathrm{~nm}$ (one photon), the signal of $\mathrm{CH}_{3} \mathrm{CO}^{+}$can be observed under the condition of heat on, while under heat off, the signal disappears. It is assured that the observed signal is definitely coming from the ionization of the acetyl radical (first channel), not from the decomposing of acetone cations (second channel). When the laser wavelength is shorter than $325 \mathrm{~nm}$, the contribution of the second channel to the observed $\mathrm{CH}_{3} \mathrm{CO}^{+}$signal becomes more and more significant; for this reason, we only studied the spectrum in the range $325-400 \mathrm{~nm}$ in this paper.

Fig. 2 shows an MPI spectrum recorded with an SR250 gated integrator when the laser wavelength was scanned continuously. This is a composite spectrum with five parts and not normalized with laser energy, but an intensity scaling based on

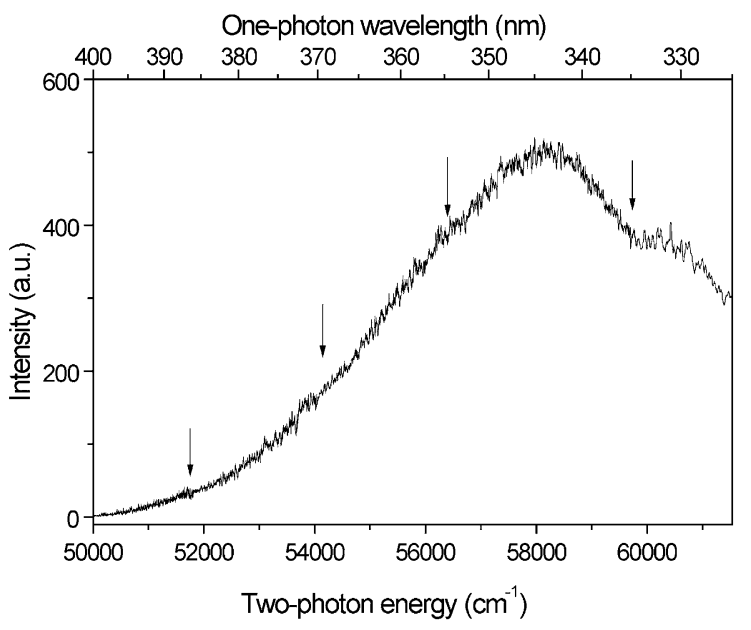

Fig. 2. Wavelength continuously scanned MPI spectrum of the acetyl radical in the range $325-400 \mathrm{~nm}$ (one photon). The spectrum is not normalized with laser power. Arrows point to the overlap position of every two neighboring dyes. 
common signals in the overlapping region of every two neighboring dyes was enforced. The spectrum is continuous and structureless, that is to say, without any vibronic structure.

In order to obtain an MPI spectrum normalized with laser energy, we measured the relative ion yield of the acetyl cations point by point in the range $325-385 \mathrm{~nm}$. In this experiment, wavelength step was $1 \mathrm{~nm}$ and laser energy on each point was controlled to be $0.85 \mathrm{~mJ}$. The relative values of ion yield were obtained by measuring the integrated intensity of the $\mathrm{CH}_{3} \mathrm{CO}^{+}$signal with the LECroy 9344 digital oscilloscope. In order to minimize the measurement error coming from the laser energy fluctuation, each value was the averaged result of three measurements, and each measurement datum was averaged from 1000 laser shots. Fig. 3 shows this spectrum as obtained point by point.

Power-dependence experiment is helpful for determining whether the acetyl radical is ionized

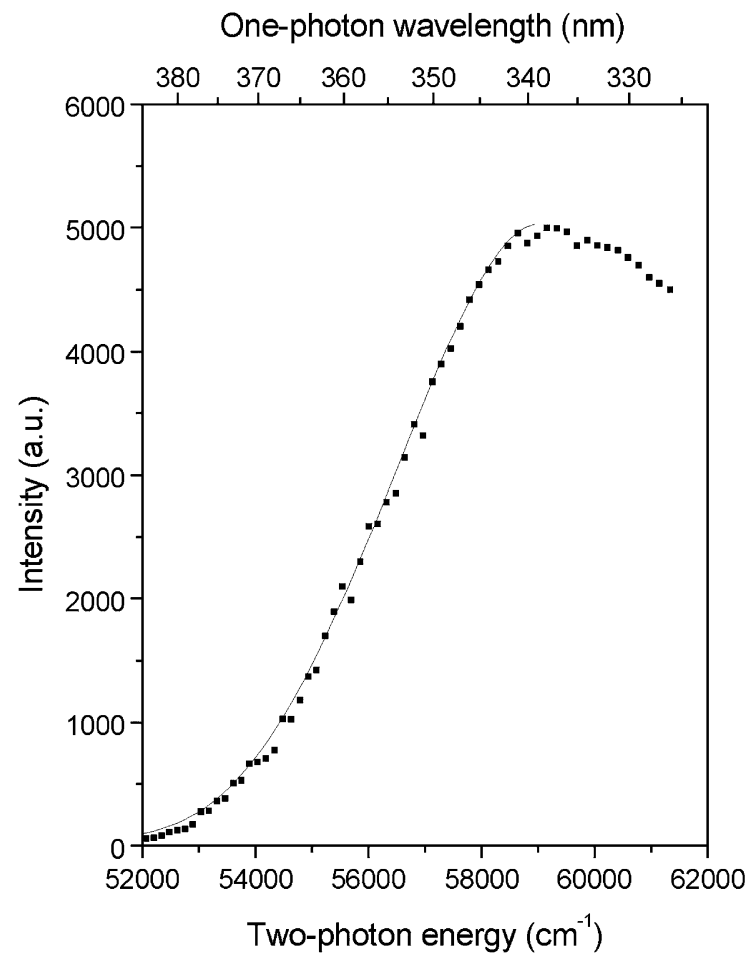

Fig. 3. MPI spectrum of the acetyl radical at $50000-61540 \mathrm{~cm}^{-1}(6.2-7.6 \mathrm{eV})$ obtained point by point at the same laser energy, $0.85 \mathrm{~mJ}$. via two-photon process. It is well known that, for MPI

$I_{\mathrm{S}} \propto P^{n}$,

where $I_{\mathrm{S}}$ is the ion yield, $P$ is the laser power, and $n$ is the power coefficient. For $2+1$ REMPI, $2 \leqslant n \leqslant 3$, the reason is optical saturation in the final ionization step. In the case of full saturation in the final ionization process, $n=2$. For ionization process via absorbing two photons simultaneously, $n=2$.

We performed a power-dependence measurement at different wavelengths. In the experiment, quartz plates were used to attenuate laser power. The power coefficients were measured to be in the range 1.9-2.3 because of measurement errors. Fig. 4 is the $\log -\log$ plot of ion yield vs laser energy excited at 370 and $340 \mathrm{~nm}$. At $370 \mathrm{~nm}, n=2.26$, and at $340 \mathrm{~nm} n=2.02$.

It is not enough to determine if the mechanism of the observed MPI spectrum in Fig. 2 is either $2+1$ REMPI or direct ionization via absorbing two photons simultaneously only according to the measured power coefficients. It is necessary to refer to the ionization potential of the acetyl radical. The ionization potential of the acetyl radical had been measured with different methods. In 1957, Reed and Brand [24] determined the IP to be $8.08 \pm 0.09 \mathrm{eV}$ with electron impact studies. In

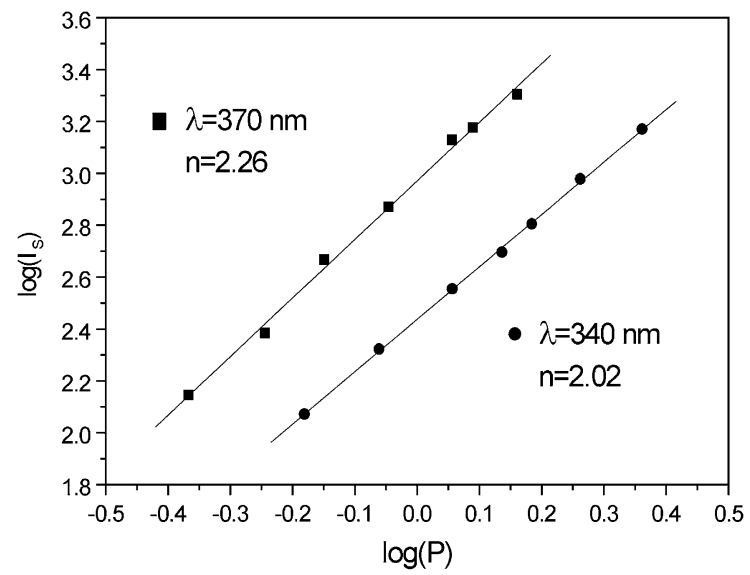

Fig. 4. Log-log plot of ion yield vs laser energy excited at 370 and $340 \mathrm{~nm}$. The power coefficients are fitted to be 2.26 and 2.02 , respectively. 
1963, Murad and Inghram [25] determined the adiabatic ionization potential to be $7.08 \mathrm{eV}$ with photoionization of aliphatic ketones; no error was reported in their measurement. On the other hand, Lias et al. [26] reported that the ionization potential of the acetyl radical was $7.0 \mathrm{eV}$.

The pattern of the MPI spectra of the acetyl radical in $50000-61540 \mathrm{~cm}^{-1}$ is similar to that of the MPI spectra of some neutral molecules such as propyne and vinyl chloride studied in our laboratory in the region of the near ionization threshold $[21,27]$. In the case of two-photon energy being higher than the ionization potential, MPI spectrum becomes continuous. According to this characteristic and referring to the reported ionization potential of the acetyl radical as well as power coefficients obtained in our experiments, we draw a conclusion that the acetyl radical is ionized via $2+1$ REMPI at $370 \mathrm{~nm}$ and is directly ionized via absorbing two photons simultaneously at 340 nm. Abou-Zied and McDonald [23] proposed a $2+1$ REMPI mechanism excited at $400 \mathrm{~nm}$ with picosecond pulse laser. Our conclusion is in agreement with theirs, if considering the same excitation wavelength.

\subsection{Adiabatic ionization potential of the acetyl radical}

Based on the previous analysis, the continuous spectral band of $59000-615400 \mathrm{~cm}^{-1}$ of the acetyl radical in Fig. 3 can be assigned to direct ionization via absorbing two photons, and therefore, the ionization potential of acetyl radical is impossible to be $8.08 \mathrm{eV}\left(65173 \mathrm{~cm}^{-1}\right)$. Using fifth-order polynomial fit the spectrum between 52000 and $59000 \mathrm{~cm}^{-1}$ and doing the first-order derivative to the fitted curve, the maximum of the derivative, $56569 \mathrm{~cm}^{-1}(7.01 \mathrm{eV})$ corresponding to the adiabatic ionization potential of the acetyl radical was obtained (see Fig. 5). Because the spectrum at $52000-59000 \mathrm{~cm}^{-1}$ was obtained point by point, the measurement error was estimated to be less than one increasing wavelength step. In our experiment, the wavelength step is $\Delta \lambda=0.5 \mathrm{~nm}$ if considering two-photon processing, and then $\Delta k_{\mathrm{IP}}=160 \mathrm{~cm}^{-1} \approx 0.02 \mathrm{eV}$. That is to say, the

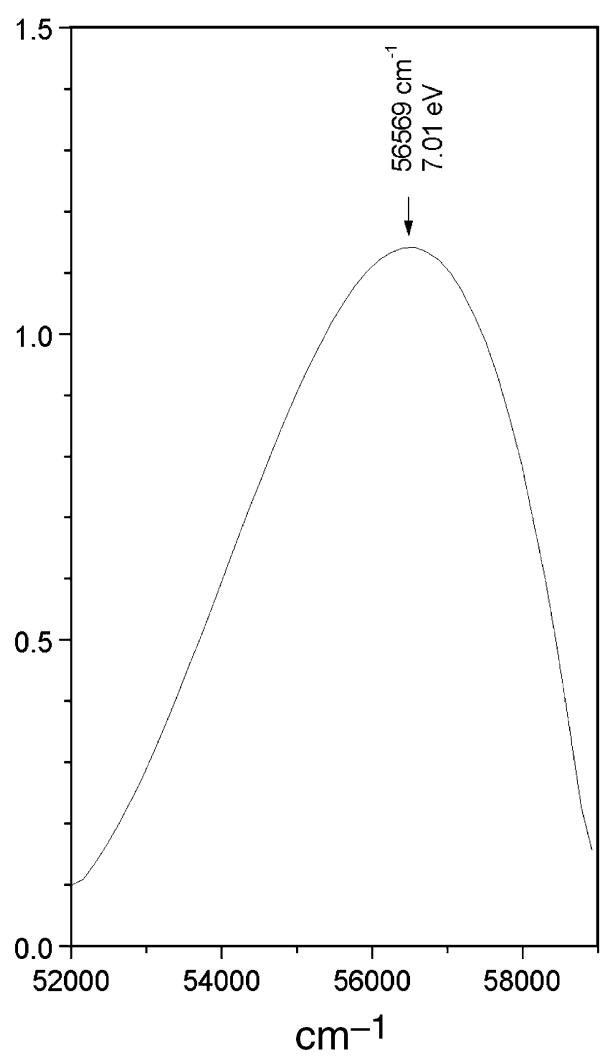

Fig. 5. Derivative of the MPI spectrum of the acetyl radical near the ionization threshold. The maximum, $56569 \mathrm{~cm}^{-1}$, is responsible for the adiabatic ionization potential.

ionization potential of the acetyl radical determined by us is IP $=7.01 \pm 0.02 \mathrm{eV}$.

Unlike allyl radical [22], no Rydberg series was observed in the MPI spectrum of the acetyl radical, and therefore the ionization potential cannot be determined with the Rydberg formula

$v=\mathrm{IP}-\frac{R}{(n-\delta)^{2}}$,

where $v$ is the term value in $\mathrm{cm}^{-1}, R$ is the Rydberg constant, and $n$ is the principal quantum number, and $\delta$ is the corresponding quantum defect. On the other hand, because the acetyl cation is stable, the zero-kinetic energy (ZEKE) technique would be the best method to determine the ionization potential of an acetyl radical accurately. 


\section{Discussion}

The continuous $2+1$ REMPI spectrum of the acetyl radical from $50000 \mathrm{~cm}^{-1}$ to the ionization threshold is impossible to have resulted from vibronic congestion of the hot acetyl radical. One interpretation is that the highly excited electronic states of the acetyl radical are metastable; it is dissociative to result in a continuous spectrum. But the dissociative rate is less than that of lowlying B state. One experimental evidence is that we try to find out $1+1$ REMPI signal in the region of 200-250 $\mathrm{nm}$ and 2 + 1 REMPI signal in the region 200-270 nm (two-photon wavelength) but we cannot observe any ionization signal of the acetyl radical under the condition of heat on. According to the theoretical calculation done by Mao et al. [14], C and D electronic states of the acetyl radical lie in the range $6.2 \mathrm{eV}$-IP, while $\mathrm{B}$ state lies in the range $200-270 \mathrm{~nm}$. According to this, a dynamical information can be achieved that $\mathrm{C}$ and $\mathrm{D}$ states are less dissociative than B state for the acetyl radical. Generally, the B, C, D states of the acetyl radical are dissociative, and without any vibronic spectral structure can be observed in UV absorption or REMPI spectra.

\section{Conclusions}

Jet-cooled acetyl radical can be produced by pyrolysis of acetone in a molecular beam and can be monitored with the MPI technique. A continuous MPI spectrum at $6.2-7.6 \mathrm{eV}$ of the radical was observed in experiment. The spectrum at $6.2 \mathrm{eV}$-IP has resulted from $2+1$ REMPI through $\mathrm{C}, \mathrm{D}$ and high-lying Rydberg states; the spectrum at IP-7.6 eV has resulted from direct two-photon ionization. Highly excited electronic states such as C, D of the acetyl radical have proved to be dissociative and metastable. In comparison, the low-lying electronic states of the radical B are very easy to dissociate. The adiabatic ionization potential of the acetyl radical is redetermined to be $7.01 \pm 0.02 \mathrm{eV}$ from the derivative of the MPI spectrum near the ionization threshold.

\section{Acknowledgements}

This work is supported by National Science Council, Taiwan and China Petroleum Corporation. Li Run-Hua is also grateful to the financial support of National Natural Science Foundation of China (Grant No. 69908007) and to the Natural Science Foundation of Guangdong Province, China (Grant No. 980278) for this project.

\section{References}

[1] W.C. Brider, Combustion Chemistry, Springer, New York, 1984.

[2] B.J. Finlayson-Pitts, J.N. Pitts Jr., Atmospheric Chemistry: Fundamentals and Experimental Techniques, Wiley, New York, 1986.

[3] M.R. Nimlos, J.A. Soderquist, G.B. Ellison, J. Am. Chem. Soc. 111 (20) (1989) 7675.

[4] J.E. Bennett, B. Mile, Trans. Faraday Soc. 67 (1971) 1587.

[5] J.S. Francisco, N.J. Abersold, Chem. Phys. Lett. 187 (1991) 354.

[6] S. Deshmukh, W.P. Hess, J. Chem. Phys. 100 (9) (1994) 6429.

[7] S. Deshmukh, J.D. Myers, S.S. Xantheas, W.P. Hess, J. Phys. Chem. 98 (1994) 12535.

[8] S. North, D.A. Blank, Y.T. Lee, Chem. Phys. Lett. 224 (1994) 38.

[9] S.W. North, D.A. Blank, J.D. Gezelter, C.A. Longfellow, Y.T. Lee, J. Chem. Phys. 102 (11) (1995) 4447.

[10] S.K. Kim, S. Pedersen, A.H. Zewail, J. Chem. Phys. 103 (1) (1995) 477.

[11] J.E. Bennett, S.C. Graham, B. Mile, Spectrochimica Acta 29A (1973) 375.

[12] M.E. Jacox, Chem. Phys. 69 (1982) 407.

[13] N.C. Baird, H.B. Kathpal, Can. J. Chem. 55 (1977) 863.

[14] W.T. Mao, Q. Li, F. Kong, M.B. Huang, Chem. Phys. Lett. 283 (1998) 114.

[15] S. Noda, K. Fueki, Z.I. Kuri, J. Chem. Phys. 49 (70) (1961) 3287.

[16] H. Adachi, N. Basco, D.G.L. Jams, Chem. Phys. Lett. 59 (1978) 502.

[17] D.A. Parkes, Chem. Phys. Lett. 77 (1981) 527.

[18] M.M. Maricq, J.J. Szente, Chem. Phys. Lett. 253 (1996) 333.

[19] H.Z. Li, Q. Li, W.T. Mao, Q.H. Zhu, F. Kong, J. Chem. Phys. 106 (14) (1997) 5943.

[20] T. Shibata, H.Y. Li, H. Katayanagi, T. Suzuki, J. Phys. Chem. A 102 (1998) 3643.

[21] J.C. Shieh, J.L. Chang, J.C. Wu, R.H. Li, A.M. Mebel, N.C. Handy, Y.T. Chen, J. Chem. Phys. 112 (17) (2000) 7384.

[22] J.C. Wu, R.H. Li, J.C. Chang, Y.T. Chen, J. Chem. Phys. 113 (17) (2000) 7286. 
[23] O.K. Abou-Zied, J.D. McDonald, J. Chem. Phys. 109 (4) (1998) 1293.

[24] R.I. Reed, J.C. Brand, J. Chem. Soc. Faraday Trans. 54 (1958) 478.

[25] E. Murad, M.G. Inghram, J. Chem. Phys. 40 (11) (1964) 3263.
[26] S.G. Lias, J.E. Bartmess, J.F. Liebman, J.L. Holmes, R.D. Levin, W.G. Mallard, J. Phys. Chem. Ref. Data (Suppl. 17) (1998) 861.

[27] J.L. Chang, J.C. Shieh, J.C. Wu, R.H. Li, Y.T. Chen, Chem. Phys. Lett. 325 (2000) 369. 